\title{
Role of the RNA polymerase trigger loop in nucleotidyl transfer, transcript cleavage, and transcriptional pausing
}

\author{
Jinwei Zhang ${ }^{1,4}$, Murali Palangat ${ }^{2}$, and Robert Landick ${ }^{2,3}$ \\ ${ }^{1}$ Department of Biomolecular Chemistry, University of Wisconsin, Madison, WI 53706 \\ ${ }^{2}$ Department of Biochemistry, University of Wisconsin, Madison, WI 53706 \\ ${ }^{3}$ Department of Bacteriology, University of Wisconsin, Madison, WI 53706
}

\section{Abstract}

The trigger loop (TL) is a polymorphous component of RNA polymerase (RNAP) that makes direct substrate contacts and promotes nucleotide addition when folded into an a-helical hairpin (trigger helices; TH). However, the roles of the TL/TH in transcript cleavage, catalysis, substrate selectivity, and pausing remain ill-defined. Based on in vitro assays of Escherichia coli RNAP bearing specific TL/TH alterations, we report that neither intrinsic nor regulator-assisted transcript cleavage of backtracked RNA requires formation of the TH. We find that the principal contribution of TH formation to rapid nucleotidyl transfer is steric alignment of the reactants, rather than acid-base catalysis, and that the TL/TH cannot be the sole contributor to substrate selectivity. The similar effects of TL/TH substitutions on pausing and nucleotide addition provide additional support for the view that $\mathrm{TH}$ formation is rate-limiting for escape from nonbacktracked pauses.

\section{Introduction}

Multisubunit RNAPs use highly conserved active-site dynamics to initiate, elongate, pause, and proofread transcription, processes that are central to gene expression in all cellular organisms 1-10. The RNAP active site catalyzes two distinct reactions, nucleotidyl transfer and RNA hydrolysis (transcript cleavage) (Fig. 1a)1,10,11. In the nucleotidyl transfer reaction, a nucleoside monophosphate (from a templated NTP substrate) is attached to the $3^{\prime}$ $\mathrm{OH}$ of the growing RNA chain, followed by release of pyrophosphate (PPi). In the reverse reaction, PPi acts as a nucleophile to shorten the RNA chain one nucleotide (nt) at a time with release of NTP. The hydrolysis reaction uses $\mathrm{OH}^{-}$instead of PPi as a nucleophile when an internal RNA phosphodiester occupies the active site. This transcript cleavage reaction can rescue RNAP from transcriptional arrest10,11, can occur by intrinsic catalysis, and can be accelerated by transcript cleavage factors like GreA/B and TFIIS10-16.

Both nucleotidyl transfer and transcript cleavage use a two- $\mathrm{Mg}^{2+}$-mediated $\mathrm{S}_{\mathrm{N}} 2$ reaction mechanism (Fig. 1a)11,17. In bacterial RNAP, $\mathrm{Mg}^{2+} \mathrm{I}\left(\mathrm{K}_{\mathrm{d}} \approx 100 \mu \mathrm{M}\right) 18$ remains bound at physiological $\mathrm{Mg}^{2+}$ concentrations, whereas $\mathrm{Mg}^{2+} \mathrm{II}\left(\mathrm{K}_{\mathrm{d}} \geq 10 \mathrm{mM}\right) 11$ must be recruited via stabilizing contacts to NTP phosphates11, to backtracked RNA10, or to acidic groups on transcript cleavage factors15. Both reactions also involve proton transfers that could be facilitated by general acid/base catalysis19,20 (Fig. 1a).

\footnotetext{
Correspondence should be addressed to R.L. (landick@bact.wisc.edu).

${ }^{4}$ Present address: Division of Basic Sciences, Fred Hutchinson Cancer Research Center, Seattle, WA 98109, USA.
} 
Folding of the TL into the TH strongly enhances the rate of nucleotidyl transfer(by up to 10,000 -fold) via substrate contacts made by the TH ( $\beta^{\prime}$ M932 to NTP base; $\beta^{\prime}$ R933 and H936 to NTP phosphates; Fig. 1b)6,8,21,22. The TL/TH is the target of lineage-specific sequence insertions (SI) like SI3 in E. coli RNAP ( $\left.\beta^{\prime} 943-1130\right)$, which forms a two-lobed domain at the entrance to the secondary channel and is connected to the TL/TH by flexible linkers 23,24 . The TH pack against a second conserved $\beta^{\prime}$ element called the bridge helix $(\mathrm{BH})$; together, the $\mathrm{TH}$ and $\mathrm{BH}$ form a metastable three-helix bundle (THB) that modulates TH-TL oscillation by virtue of carefully balanced side-chain contacts within the THB, to the NTP substrate, to the RNA:DNA hybrid, and to other parts of RNAP (Fig. 1c.). Blocking TH formation by substituting two Pro residues for adjacent residues not involved in key contacts inhibits nucleotidyl transfer without affecting NTP binding $\left(\beta^{\prime} \mathrm{L} 930, \mathrm{~T} 931 \rightarrow \mathrm{PP}\right.$, $\mathrm{TH}^{\mathrm{PP}}$; Fig. 1a,b)6,22. The essentiality of the TL/TH to function of multisubunit RNAPs is highlighted by several naturally evolved inhibitors that bind the TL and prevent TH formation (e.g., streptolydigin and a-amanitin)6,25-28.

It has been uncertain whether TH formation is required for transcript cleavage, how THNTP contacts promote rapid nucleotidyl transfer, whether they dictate selection of NTPs vs. dNTPs, and how the TL-TH transition is involved in transcriptional pausing. TH contacts are suggested to facilitate acid/base catalysis during nucleotidyl transfer via interaction of $\beta$ 'H936 (Rpo21p/Rpb1p H1085 in S. cerevisiae RNAPII) with the $\beta$ phosphate8,20. Based on an H1085Y substitution in $S$. cerevisiae RNAPII, the TL is proposed to make a 5- to 10-fold contribution to an overall $\sim 500$ - to 5000 -fold discrimination of NTP over $2^{\prime} \mathrm{dNTP} 8,26$. It is unclear if this represents the partial effect of one residue in a much greater overall contribution of the TH to substrate selectivity, or if formation of the THB is not essential for substrate selection. Finally, inhibition of THB formation has been proposed to be the mechanistic basis of elemental pauses22. The elemental pause is an initially formed, nonbacktracked state that can be stabilized in longer-lived pauses by nascent RNA hairpins or backtracking22, and likely explains ubiquitous, nonbacktracked pausing detected in single-molecule transcription experiments 29,30 . However the role of the TL/TH in the elemental pause remains ill-defined.

To address these questions, we used specific biochemical assays of RNAP active site functions to test mutant RNAPs with different TL/TH alterations.

\section{Results}

\section{Role of THB in nucleotidyl transfer and transcript hydrolysis}

We previously reported that the $\mathrm{TH}^{\mathrm{PP}}$ substitutions or outright deletion of the TL dramatically inhibit nucleotidyl transfer without affecting NTP binding (for both nucleotide addition and pyrophosphorolysis)6,22 (Fig. 1a,b; compare WT and $\mathrm{TH}^{\mathrm{PP}}$ ). However, it remains unknown if internal transcript cleavage requires THB formation (Fig. 1a, right panel).

To test the role of the THB in internal transcript cleavage, we used a reconstituted elongation complex (EC) locked in a 2-nt backtracked configuration by noncomplementarity of the RNA outside the 9 bp RNA:DNA hybrid and by noncomplementarity of the nontemplate and template DNA strands within the transcription bubble (EC ${ }^{\mathrm{U}-2}$; Fig. 2a). Because the scaffold in $\mathrm{EC}^{\mathrm{U}-2}$ locks the translocation register, this assay should reveal hydrolysis rates unaffected by sliding of RNAP. $\mathrm{EC}^{\mathrm{U}-2}$ releases the 3'-terminal pUpUpU trimer, shortening the transcript from $16 \mathrm{nt}$ to $13 \mathrm{nt}$, upon incubation with $20 \mathrm{mM} \mathrm{Mg}^{2+}$ at $\mathrm{pH}$ 9.022, with or without added GreB (Fig. 2b). Strikingly, either deleting the TL or blocking THB formation with the $\mathrm{TH}^{\mathrm{PP}}$ substitutions had little effect on the rate of intrinsic transcript cleavage (Figs. 2b,c). To verify that the TL/TH did not affect $\mathrm{Mg}^{2+}$ II-binding, we measured 
the rate of transcript cleavage by $\triangle \mathrm{TL}$ RNAP from 1 to $80 \mathrm{mM} \mathrm{Mg}^{2+}$ and observed cleavage rates equivalent to wild-type RNAP (Fig. 2d,e). We conclude that THB formation is not required for intrinsic cleavage of this 2-nt backtracked RNA by E. coli RNAP, in contrast to its key function in nucleotidyl transfer.

\section{GreB requires SI3, but not the THB, to stimulate RNA hydrolysis}

The rate of intrinsic transcript cleavage by RNAP can be greatly stimulated by accessory proteins that transiently interact with RNAP (e.g., GreB; Fig. 2b). These regulators (the paralogs GreA and GreB in bacteria and the structurally unrelated TFIIS in eukaryotes) are proposed to stabilize $\mathrm{Mg}^{2+} \mathrm{II}$ or $\mathrm{OH}^{-}$via invariant acidic residues that are inserted immediately adjacent to the RNAP active site (D41 and E44 of GreB)12,13,15. However, the folded TH creates an extensive barrier that shields the catalytic center from the secondary channel during catalysis6,8 and would appear to prevent GreB access to the active site. To test whether GreB action requires $\mathrm{TH}$ formation, we asked if the $\mathrm{TH}^{\mathrm{PP}}$ substitutions would affect either GreB binding or cleavage stimulation in $\mathrm{EC}^{\mathrm{U}-2}$. At either 1 $\mu \mathrm{M}$ (Fig. 2b) or $10 \mu \mathrm{M}$ (Fig. 3a), GreB had approximately the same effect on transcript cleavage in either wild-type or $\mathrm{TH}^{\mathrm{PP}} \mathrm{EC}^{\mathrm{U}-2}$. Further, the concentration of GreB required for half-maximal stimulation of transcript cleavage was also similar $\left(210 \mathrm{nM}\right.$ for $\mathrm{TH}^{\mathrm{PP}}$ compared to $360 \mathrm{nM}$ for wild type; Fig. 3b). However, when either the entire TL/TH or just the SI3 domain was deleted, GreB was unable to stimulate transcript cleavage (Fig. 3 and data not shown). We conclude that, like intrinsic transcript hydrolysis, stimulation of hydrolysis by GreB does not require TH formation. Rather, GreB appears to act on RNAP containing an unfolded TL, thus avoiding steric clash with the TH (Supplementary Fig. 1). However, SI3 is required for GreB function in E. coli RNAP, either because GreB contacts SI3 or because SI3 facilitates GreB binding or action.

\section{THB formation is not required for selection of NTP over 2 'dNTP}

In RNAPII, the TH are proposed to make a 5- to 10 -fold contribution to an overall $\sim 500$ - to 5000 -fold selectivity of NTP relative to 2'dNTP26. However, these estimates are based on the effects on discrimination of an H1085Y substitution in $S$. cerevisiae RNAPII that may not eliminate all contributions of the TH. To investigate the role of the TH in substrate section by E. coli RNAP, we tested wild-type and $\triangle$ TL RNAPs for ATP/2'dATP selectivity using a single-nucleotide addition assay designed to detect misincorporation by changes in electrophoretic mobility of a 10-nt product RNA2 (Fig. 4a). Use of this EC ${ }^{\mathrm{G} 9}$ template allows direct detection of AMP or 2'dAMP incorporation as products of different mobility (Fig. 4a), and thus avoids confusion from scavenging of trace ATP contamination2.

Although we were unable to measure the wild-type selectivity for ATP/2'dATP on this template owing to the rapid addition of AMP at saturating ATP concentration, wild-type selectivity of ATP/2'dATP has been estimated previously as $~ 1000$ based on the substrate concentrations required to achieve $50 \%$ incorporation in $2 \mathrm{~min}$ at $37^{\circ} \mathrm{C} 31$. (This is the same assay used by Kaplan et al.26 to estimate NTP incorporation rates and NTP/2'dNTP selectivity for RNAPII). We confirmed the $\sim 1000$-fold selectivity of wild-type ATP/2'dATP using this assay, but the much longer time scale required for $\triangle \mathrm{TL}$ RNAP precluded direct comparison (Supplementary Fig. 2).

Thus, for $\triangle \mathrm{TL}$ RNAP, we measured the apparent substrate affinity $K_{\mathrm{NTP}}$ app and the apparent maximal turnover rate $k_{\text {pol }}^{\text {app }}$ for both ATP and 2'dATP (Fig. 4b). This yielded $k_{\mathrm{pol}}$ app/ $\mathrm{K}_{\mathrm{NTP}}$ app of $0.011 \mathrm{~min}^{-1} \mu \mathrm{M}^{-1}$ for ATP and $0.00011 \mathrm{~min}^{-1} \mu \mathrm{M}^{-1}$ for $2^{\prime} \mathrm{dATP}$, equivalent to a selectivity of $\sim 100$ for ATP vs. dATP (Fig. $4 \mathrm{c}$ ). We conclude that THB formation at a minimum is not the sole mechanism for discrimination between NTP and dNTP substrates. 
We also examined misincorporation of non-cognate NTPs by $\triangle$ TL RNAP and found even greater discrimination than against cognate 2 'dNTPs. At $\sim 10 \mathrm{mM}$ NTPs, $\triangle$ TL RNAP needed $\sim 1$ minute to incorporate a correct ATP, but could only incorporate low levels $(<10 \%)$ of non-cognate CTP, UTP, or GTP, even after hours of incubation (data not shown). These slow misincorporation rates precluded accurate measurement since RNAP may become inactive upon prolonged incubation.

These results are consistent with a view that other components of the catalytic center, such as $\beta^{\prime} \mathrm{R} 425$ and $\beta^{\prime} \mathrm{N} 458$ in $E$. coli RNAP, make important contacts responsible for selection of NTPs over $2^{\prime}$ dNTPs 31 . However, they also are consistent with the proposed role of TH formation in kinetic discrimination against dNTPs (as opposed to a direct binding contact), with about the same overall magnitude as reported for $S$. cerevisiae RNAPII based on the effects of single amino-acid substitutions and a-amanitin.26 In other words, for both $E$. coli RNAP and $S$. cerevisiae RNAPII, contacts specific to formation of the THB during catalysis, rather than to substrate binding, contribute a factor of $\sim 10$ to an overall $\sim 1000$-fold selectivity of NTPs vs. 2'dNTPs.

\section{THB contribution to catalysis and nonbacktracked pause escape}

To investigate the role of the TH in nucleotidyl transfer, we next assayed a collection of mutant RNAPs carrying single amino-acid substitutions in the TH (Fig. 5a,b) using three different nucleotidyl transfer reactions: rapid nucleotide addition in $\mathrm{EC}^{\mathrm{A} 14}$, pyrophosphorolysis in $\mathrm{EC}^{\mathrm{U} 14}$, and escape from a nonbacktracked, paused transcription complex (PTC ${ }^{\mathrm{U} 2}$; Supplementary Fig. 3). Both rapid nucleotide addition and pyrophosphorolysis should be slowed by substitutions that inhibit THB formation, that remove nucleotide contacts that stabilize the transition state, or that remove functional groups that participate in general acid/base catalysis (e.g., $\mathrm{H} 936$ as proposed by Wang et al.8 and Castro et al.20). Both reactions might be accelerated by substitutions that increase the rate at which the open TL converts to the THB (e.g., RPB1 E1103G)2,32. Pause escape in $\mathrm{PTC}^{\mathrm{U} 29}$ appears to be rate-limited by THB formation from a "paused" TL conformation. This paused state is thought to allow translocation and NTP binding, but to inhibit THB formation22. This model predicts that TL substitutions should affect rapid nucleotidyl transfer and pause escape similarly. Effects unique to the pause state might give insight into the nature of the paused TL conformation.

Among the TL substitutions assayed, the magnitudes of effects on the three different reactions ranged from 2 -fold to 70 -fold and were well correlated (Fig. 5c). This result is consistent with the view that THB formation is similarly rate-limiting for all three reactions. Substitutions in residues that contact reaction substrates (M932 and H936) or stabilize the THB (T934) had the largest effects. Notably, substitution of M932, which is thought to stack on the nucleotide ring (Fig. 1a), and of T934 had greater effect than substitution of H936, which has been proposed to mediate acid/base catalysis8,20 (Fig. 5c). Hence, TH steric contacts appear more important than a TH role in acid/base catalysis. (The effect of M932A might also reflect effects on the recently proposed wedged-TL state25.) Stabilization of the THB with a G1136S substitution increased the rate of both pyrophosphorolysis and pause escape, consistent with previous observations 36 . Taken together, these results suggest the magnitude of TL-substitution effects is determined principally by their effects on NTP contacts or THB formation. Further, THB formation is similarly rate-limiting for nucleotidyl transfer and for escape from a nonbacktracked pause. supporting the proposed persistence of the elemental pause state until THB formation22,33,34. 


\section{Substrate positioning versus acid/base catalysis by the THB}

The TH have been proposed to contribute to nucleotidyl transfer by assisting acid/base catalysis, specifically transfer of a proton to the NTP $\beta$ phosphate by H936 (H1085 in $S$. cerevisiae RNAPII)8,20. Although the relatively small effect of the H936A substitution is at odds with this idea (4-8X effect in Fig. 5c vs. 12000X total effect of the TH), it is possible that R933 might substitute for this role when H936 is absent (this Arg is conserved in bacterial RNAP, but is an Asn in RNAPII). An Arg residue acts as a general acid/base catalyst in rat DNA polymerase $\beta 35$. To test this idea, we created and assayed an R933A,H936A double mutant RNAP. The effects of the double substitution ranged from 13-24 fold and, with the exception of pause escape, were approximately additive relative to the individual substitutions, consistent with each making an independent contribution to stabilizing the reaction transition state (Fig. 5c). (The non-additive effect on pause escape may reflect synergistic effects of the substitutions on the paused conformation of the TL.) Thus, acid-base catalysis by either R936 or R933 contributes at most $0.1 \%$ of the overall effect of the TH on nucleotidyl transfer ( 25X of a 12000X effect; Fig. 5c). Thus, the primary THB function in nucleotidyl transfer appears to be steric; via both van der Waals and electrostatic contacts (Fig. 1c, blue and red dotted lines, respectively), the THB positions the reactants for $\mathrm{S}_{\mathrm{N}} 2$ catalysis.

\section{Discussion}

Our results yield four central findings. First, neither intrinsic nor GreB-assisted hydrolysis reactions require folding of the TL/BH into the THB. Second, the THB promotes nucleotidyl transfer principally through side-chain contacts that stabilize the transition state; acid/base catalysis by R933 or H936 plays at most a lesser role. Third, formation of the THB is not the principal mechanism for substrate selectivity by RNAP. Finally, THB formation appears to be similarly rate-limiting for both rapid nucleotidyl transfer and escape from a nonbacktracked pause.

\section{Role of the TL in transcript cleavage}

Catalysis of transcript cleavage by RNAP without THB formation when the scaffold is locked against translocation makes both chemical and structural sense. From a chemical perspective, the stronger $\mathrm{OH}^{-}$nucleophile will require less energetic assistance to achieve the transition state than pyrophosphate. By requiring a backtracked nucleotide, an accessory factor (GreA/B or TFIIS), or both for $\mathrm{Mg}^{2+}$-binding at physiological concentrations10,15, the RNAP active site has evolved to preclude hydrolysis of correctly incorporated NMPs. From a structural perspective, both 2-nt backtracked RNA and cleavage-factor binding appear to be sterically incompatible with formation of the THB12,13,15,16 (Fig. 6 and Supplementary Fig. 1). Thus, by adapting the secondary channel to accommodate backtracked RNA and cleavage-factor binding in the open TL conformation, and formation of the THB upon correct NTP binding in the A site, RNAP has evolved to possess a dualfunction active site with different properties for nucleotidyl transfer and transcript hydrolysis11. The metastable THB also allows for rapid switching of the active site between a processive nucleotidyl transferase featuring cognate-NTP-induced THB formation (Fig. 6, top row) and either intrinsic or cleavage-factor stimulated cleavage of backtracked RNAs that promote TL relaxation (Fig. 6, bottom row).

We note that our findings apply to transcript cleavage on this locked scaffold by E. coli RNAP. A significant role for TL residues, though not necessarily via THB formation, has been found for intrinsic cleavage by $T$. aquaticus and $E$. coli RNAP using scaffolds with different sequence, a 10-bp RNA-DNA hybrid, and fully complementary DNA strands10 (Y. Yuzenkova and N. Zenkin, pers. comm.). 


\section{Role of the TL in nucleotidyl transfer}

Because substrate NTP binds in the active site prior to THB formation, TH-substrate contacts contribute to catalysis but not to substrate affinity6. THB formation either creates an environment in which a subsequent catalytic event occurs involving more modest shifts in residue positions, or it directly stabilizes the transition state for nucleotidyl transfer (Fig. 6 , top row). Time-resolved measurements of THB formation and catalysis will be required to distinguish these two possibilities.

Regardless, the pattern of effects on nucleotidyl transfer of TL substitutions reported here and elsewhere verifies that formation of the THB is rate-limiting for catalysis (Figs. 5 and 6)2,21,26,36. Substitutions that should stabilize the THB increase the rate of catalysis, whereas substitutions that should destabilize the THB decrease the rate of catalysis. For instance, T928A, T931A, and T934A disrupt THB-stabilizing contacts and slow nucleotidyl transfer, even though they make no substrate contacts (Fig. 5b). Conversely substitutions like G1136S in E. coli RNAP, E1103G in S. cerevisiae RNAPII2,32, or those at G72 or I98 in $M$. jannaschii RNAP21, which stabilize the THB or disrupt contacts of the unfolded TL, increase the rate of nucleotidyl transfer (Fig. 5b)36.

Our results further suggest that the major contribution of TH to catalysis is via substratepositioning TH contacts, rather than by mediating acid/base catalysis, as had been proposed based on a H1085- $\beta$ phosphate contact observed in the $S$. cerevisiae RNAPII crystal structure8,20. The relatively modest, additive effect of the R933A, H936A double substitution (Fig. 5c) establishes that acid/base catalysis by neither the orthologous $\mathrm{H} 936$ nor a nearby Arg conserved in bacteria but not eukaryotes is required for the major effect of the $\mathrm{TH}$ on catalysis.

We emphasize that our findings do not exclude participation of R933 or H936 in acid/base catalysis; they merely establish that this is not the major contribution of the THB to catalysis. A complete exploration of the effects of $\mathrm{pH}$ on catalysis is needed to discern whether they participate in acid/base catalysis20. However, we note that a bacterial EC/NTP structure identified a well-defined water molecule close to the $\beta$ and $\gamma$ phosphates that could function as a general acid in catalysis6.

\section{Role of the TL in substrate selectivity}

Our results suggest that $\triangle$ TL RNAP robustly distinguishes NTPs and $2^{\prime}$ dNTPs (by $\sim 100$ fold, Fig. 4b,c), suggesting that a TL-independent mechanism of $2^{\prime}-\mathrm{OH}$ recognition must exist. This mechanism likely involves NTP $2^{\prime} \mathrm{OH}$ contacts to $\beta^{\prime} \mathrm{R} 425$ or $\beta^{\prime} \mathrm{N} 4586,31$. Most of the 100-fold selectivity against 2 'dATP in $\triangle$ TL RNAP occurs kinetically, with $\sim 27$-fold effect on the catalytic rate and only $\sim 4$ fold on apparent substrate affinity. This result is consistent with the proposal for RNAPII that most of the $2^{\prime}$ dNTP discrimination occurs kinetically at the catalysis step 8,26 .

We emphasize that an absence of direct THB/2 ${ }^{\prime} \mathrm{OH}$ contacts is nevertheless consistent with a kinetic role of THB formation in selectivity. The overall rate of nucleotide addition in $\triangle \mathrm{TL}$ RNAP is slow and the contributions of other substrate contacts to the $\Delta \mathrm{G}^{\ddagger}$ of the transition state will be magnified. Thus, substitutions that slow transcription by destabilizing the THB can increase fidelity (e.g., I1134V in E. coli RNAP36). Conversely, substitutions that accelerate transcription by stabilizing the THB can decrease fidelity (e.g, G1136S in $E$. coli RNAP36 and E1103G in S. cerevisiae RNAPII2,32). Although the NTP discriminating contacts may lie elsewhere in the A site (including the template base), increasing THBstabilization of the transition state will lessen the ability of these contacts to select the correct substrate. In other words, the metastable THB creates a space that is most stably occupied by a proper $\mathrm{dN}-\mathrm{rN}$ base pair; making the THB more stable will increase the rate at 
which suboptimally shaped base-pairs undergo nucleotidyl transfer, resulting in increased misincorporation.

\section{Role of the TL in pausing}

Our current view is that formation of the elemental pause results from a rearrangement of the network of contacts in ECs that connect the active site, BH, TL, RNA:DNA hybrid, RNA exit channel, clamp domain, and downstream DNA. From this initial state, pausing can be modulated by regulators or by additional interactions within the EC that either stabilize the elemental paused state or lead to further rearrangements (e.g., backtracking of RNAP). At the his pause site, this rearrangement is stabilized by a nascent RNA hairpin and occurs without backtracking by RNAP; rather, it results in trapping the TL in a state that inhibits THB formation, but not translocation or substrate binding22. Multiple findings support this view: (i) the lack of force effects on ubiquitous pauses in single-molecule experiments30; (ii) direct observation of the absence of backtracking in the paused state22,29; (iii) the ability of the paused TL state to prevent streptolydigin action22; (iv) the inability of the paused state to undergo pyrophosphorolysis37; $(V)$ the ability of sequence changes in DNA or RNA far from the active site to affect pausing in an additive fashion38; ( vi) the recent observation in an RNAPII crystal structure 39 of the frayed 3' nt conformation predicted to occur in the elemental pause22; and (vii) the recent report that a nonbacktracked pause state persists after translocation 33 .

In this model, the rate-limiting step for escape from nonbacktracked pauses is formation of the THB after NTP binding (Fig. 6, middle row)22. Alternatively, the TL could interact specifically with the paused RNA 3' nt and the rate-limiting step for pause escape could be disruption of this interaction and reversion of the paused state to the active elongation pathway prior to translocation and NTP binding. Our results strongly favor the first view. If pause-specific TL-RNA 3'-nt interaction were rate-limiting for pause escape, the substitutions with greatest effects on this interaction should be different than the substitutions with greatest effects on active elongation (compare the middle panel to the right panel, Fig. 5b). The fact that the TL substitutions exhibit similar effects on both pause escape and nucleotidyl transfer argues that the $\mathrm{TL} \rightarrow \mathrm{THB}$ transition plays similar ratelimiting roles in both events, even though the rate of the transition is much slower from the pause state because the paused conformation inhibits TL folding.

\section{Overall role of the TL/TH-BH module in regulation of RNAP}

The TL/BH module, which oscillates between an open TL form and the THB, is the key moving part in the RNAP catalytic machine. Opening and closing of the THB is energetically balanced with sets of TH, TH-BH, TH-RNAP, BH-RNAP, TH-substrate, and $\mathrm{BH}$-substrate contacts as well as helix-stabilizing (Ala) and helix-destabilizing residues (Pro and Gly) that make the THB metastable $2,8,21,22,26$. This metastability ensures that the TLBH module can oscillate rapidly for fast transcript elongation, but the entire network of contacts appears to have evolved for an intermediate rate of nucleotide addition21. This allows transcription factors (such as GreB, NusA, NusG, and numerous eukaryotic elongation regulators) to control RNAP through distal contacts that indirectly increase or decrease the rate of THB formation, but it also makes the TL-BH module susceptible to jamming as appears to happen when RNAP enters a paused state (Fig. 6, middle row) or encounters antibiotics and toxins. A thorough elucidation of the complete network of contacts, which extend throughout RNAP, will be required to understand the molecular details of regulation in this complex molecular machine. 


\section{Supplementary Material}

Refer to Web version on PubMed Central for supplementary material.

\section{Acknowledgments}

We thank members of the Landick laboratory for many suggestions during the course of this work and anonymous reviewers for excellent critique of the manuscript. This work was supported by NIH grant GM38660 to R.L.

\section{References}

1. Iyer LM, Koonin EV, Aravind L. Evolution of bacterial RNA polymerase: implications for largescale bacterial phylogeny, domain accretion, and horizontal gene transfer. Gene. 2004; 335:73-88. [PubMed: 15194191]

2. Kireeva ML, et al. Transient reversal of RNA polymerase II active site closing controls fidelity of transcription elongation. Mol Cell. 2008; 30:557-566. [PubMed: 18538654]

3. Landick R. Active-site dynamics in RNA polymerases. Cell. 2004; 116:351-353. [PubMed: 15016367]

4. Rhodes G, Chamberlin MJ. Ribonucleic acid chain elongation by Escherichia coli ribonucleic acid polymerase: Isolation of ternary complexes and the kinetics of elongation. J. Biol. Chem. 1974; 249:6675-6683. [PubMed: 4608711]

5. Richardson JP. Loading Rho to terminate transcription. Cell. 2003; 114:157-159. [PubMed: 12887917]

6. Vassylyev D, et al. Structural basis for substrate loading in bacterial RNA polymerase. Nature. 2007; 448:157-162. [PubMed: 17581590]

7. Vassylyev DG, et al. Crystal structure of a bacterial RNA polymerase holoenzyme at $2.6 \mathrm{~A}$ resolution. Nature. 2002; 417:712-719. [PubMed: 12000971]

8. Wang D, Bushnell DA, Westover KD, Kaplan CD, Kornberg RD. Structural basis of transcription: role of the trigger loop in substrate specificity and catalysis. Cell. 2006; 127:941-954. [PubMed: 17129781]

9. Yarnell WS, Roberts JW. Mechanism of instrinsic termination and antitermination. Science. 1999; 284:611-615. [PubMed: 10213678]

10. Zenkin N, Yuzenkova Y, Severinov K. Transcript-assisted transcriptional proofreading. Science. 2006; 313:518-520. [PubMed: 16873663]

11. Sosunov V, et al. Unified two-metal mechanism of RNA synthesis and degradation by RNA polymerase. Embo J. 2003; 22:2234-2244. [PubMed: 12727889]

12. Kettenberger H, Armache KJ, Cramer P. Complete RNA polymerase II elongation complex structure and its interactions with NTP and TFIIS. Mol Cell. 2004; 16:955-965. [PubMed: 15610738]

13. Opalka N, et al. Structure and function of the transcription elongation factor GreB Bound to bacterial RNA polymerase. Cell. 2003; 114:335-345. [PubMed: 12914698]

14. Orlova M, Newlands J, Das A, Goldfarb A, Borukhov S. Intrinsic transcript cleavage activity of RNA polymerase. Proc Natl Acad Sci U S A. 1995; 92:4596-4600. [PubMed: 7538676]

15. Sosunova E, et al. Donation of catalytic residues to RNA polymerase active center by transcription factor Gre. Proc Natl Acad Sci U S A. 2003; 100:15469-15474. [PubMed: 14668436]

16. Wang D, et al. Structural basis of transcription: backtracked RNA polymerase II at 3.4 angstrom resolution. Science. 2009; 324:1203-1206. [PubMed: 19478184]

17. Steitz TA. A mechanism for all polymerases. Nature. 1998; 391:231-232. [PubMed: 9440683]

18. Mustaev A, et al. Modular organization of the catalytic center of RNA polymerase. Proc Natl Acad Sci U S A. 1997; 94:6641-6645. [PubMed: 9192618]

19. Castro $\mathrm{C}$, et al. Two proton transfers in the transition state for nucleotidyl transfer catalyzed by RNA- and DNA-dependent RNA and DNA polymerases. Proc Natl Acad Sci U S A. 2007; 104:4267-4272. [PubMed: 17360513] 
20. Castro C, et al. Nucleic acid polymerases use a general acid for nucleotidyl transfer. Nat Struct Mol Biol. 2009; 16:212-218. [PubMed: 19151724]

21. Tan L, Wiesler S, Trzaska D, Carney HC, Weinzierl RO. Bridge helix and trigger loop perturbations generate superactive RNA polymerases. J Biol. 2008; 7:40-54. [PubMed: 19055851]

22. Toulokhonov I, Zhang J, Palangat M, Landick R. A central role of the RNA polymerase trigger loop in active-site rearrangement during transcriptional pausing. Mol Cell. 2007; 27:406-419. [PubMed: 17679091]

23. Artsimovitch I, Svetlov V, Murakami K, Landick R. Co-overexpression of E. coli RNA polymerase subunits allows isolation and analysis of mutant enzymes lacking lineage-specific sequence insertions. J. Biol. Chem. 2003; 278:12344-12355. [PubMed: 12511572]

24. Chlenov M, et al. Structure and Function of Lineage-specific Sequence Insertions in the Bacterial RNA Polymerase $\beta^{\prime}$ Subunit. J. Mol. Biol. 2005; 353:138-154. [PubMed: 16154587]

25. Brueckner F, Cramer P. Structural basis of transcription inhibition by alpha-amanitin and implications for RNA polymerase II translocation. Nat Struct Mol Biol. 2008; 15:811-818. [PubMed: 18552824]

26. Kaplan CD, Larsson KM, Kornberg RD. The RNA polymerase II trigger loop functions in substrate selection and is directly targeted by alpha-amanitin. Mol Cell. 2008; 30:547-556. [PubMed: 18538653]

27. Temiakov D, et al. Structural basis of transcription inhibition by antibiotic streptolydigin. Mol Cell. 2005; 19:655-666. [PubMed: 16167380]

28. Tuske S, et al. Inhibition of bacterial RNA polymerase by streptolydigin: stabilization of a straightbridge-helix active-center conformation. Cell. 2005; 122:541-552. [PubMed: 16122422]

29. Herbert KM, et al. Sequence-resolved detection of pausing by single RNA polymerase molecules. Cell. 2006; 125:1083-1094. [PubMed: 16777599]

30. Neuman KC, Abbondanzieri EA, Landick R, Gelles J, Block SM. Ubiquitous transcriptional pausing is independent of RNA polymerase backtracking. Cell. 2003; 115:437-447. [PubMed: 14622598]

31. Svetlov V, Vassylyev DG, Artsimovitch I. Discrimination against deoxyribonucleotide substrates by bacterial RNA polymerase. J Biol Chem. 2004; 279:38087-38090. [PubMed: 15262972]

32. Malagon F, et al. Mutations in the Saccharomyces cerevisiae RPB1 gene conferring hypersensitivity to 6-azauracil. Genetics. 2006; 172:2201-2209. [PubMed: 16510790]

33. Kireeva ML, Kashlev M. Mechanism of sequence-specific pausing of bacterial RNA polymerase. Proc Natl Acad Sci U S A. 2009; 106:8900-8905. [PubMed: 19416863]

34. Landick R. Transcriptional pausing without backtracking. Proc Natl Acad Sci U S A. 2009; 106:8797-8798. [PubMed: 19470457]

35. Kraynov VS, Showalter AK, Liu J, Zhong X, Tsai MD. DNA polymerase beta: contributions of template-positioning and dNTP triphosphate-binding residues to catalysis and fidelity. Biochemistry. 2000; 39:16008-16015. [PubMed: 11123928]

36. Bar-Nahum G, et al. A ratchet mechanism of transcription elongation and its control. Cell. 2005; 120:183-193. [PubMed: 15680325]

37. Toulokhonov I, Landick R. The flap domain is required for pause RNA hairpin inhibition of catalysis by RNA polymerase and can modulate intrinsic termination. Mol Cell. 2003; 12:11251136. [PubMed: 14636572]

38. Chan CL, Wang D, Landick R. Multiple interactions stabilize a single paused transcription intermediate in which hairpin to 3 ' end spacing distinguishes pause and termination pathways. J Mol Biol. 1997; 268:54-68. [PubMed: 9149141]

39. Sydow JF, et al. Structural basis of transcription: mismatch-specific fidelity mechanisms and paused RNA polymerase II with frayed RNA. Mol Cell. 2009; 34:710-721. [PubMed: 19560423]

40. Vassylyev DG, Vassylyeva MN, Perederina A, Tahirov TH, Artsimovitch I. Structural basis for transcription elongation by bacterial RNA polymerase. Nature. 2007; 448:157-162. [PubMed: 17581590]

41. Wang D, et al. Discontinuous movements of DNA and RNA in RNA polymerase accompany formation of a paused transcription complex. Cell. 1995; 81:341-350. [PubMed: 7537637] 
42. Kyzer S, Ha KS, Landick R, Palangat M. Direct versus limited-step reconstitution reveals key features of an RNA hairpin-stabilized paused transcription complex. J Biol Chem. 2007; 282:19020-19028. [PubMed: 17502377]

43. Sidorenkov I, Komissarova N, Kashlev M. Crucial role of the RNA:DNA hybrid in the processivity of transcription. Mol. Cell. 1998; 2:55-64. [PubMed: 9702191] 
a

Nucleotidyl transfer

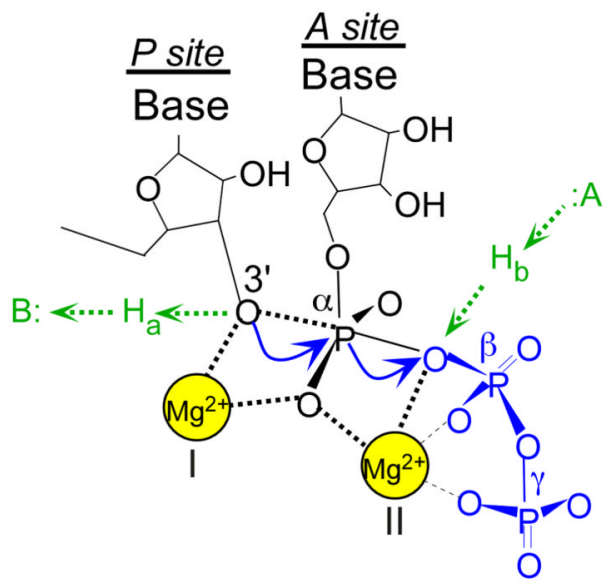

Hydrolysis (cleavage)

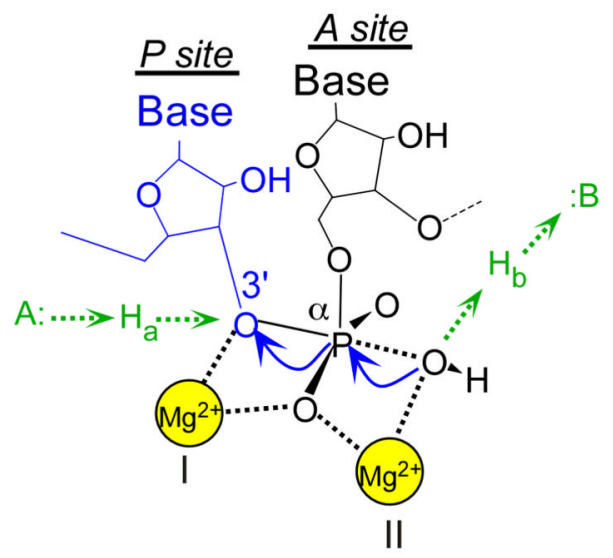

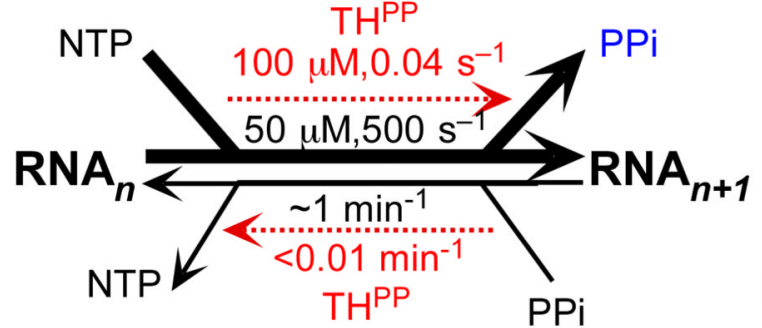

b
C

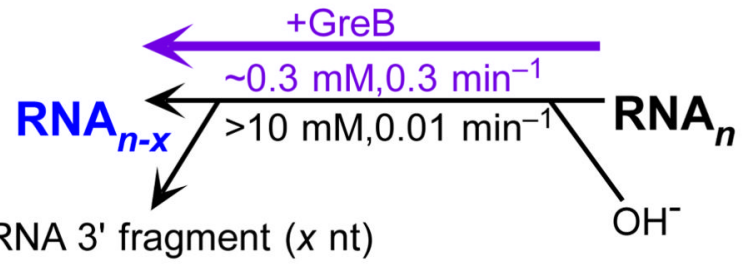

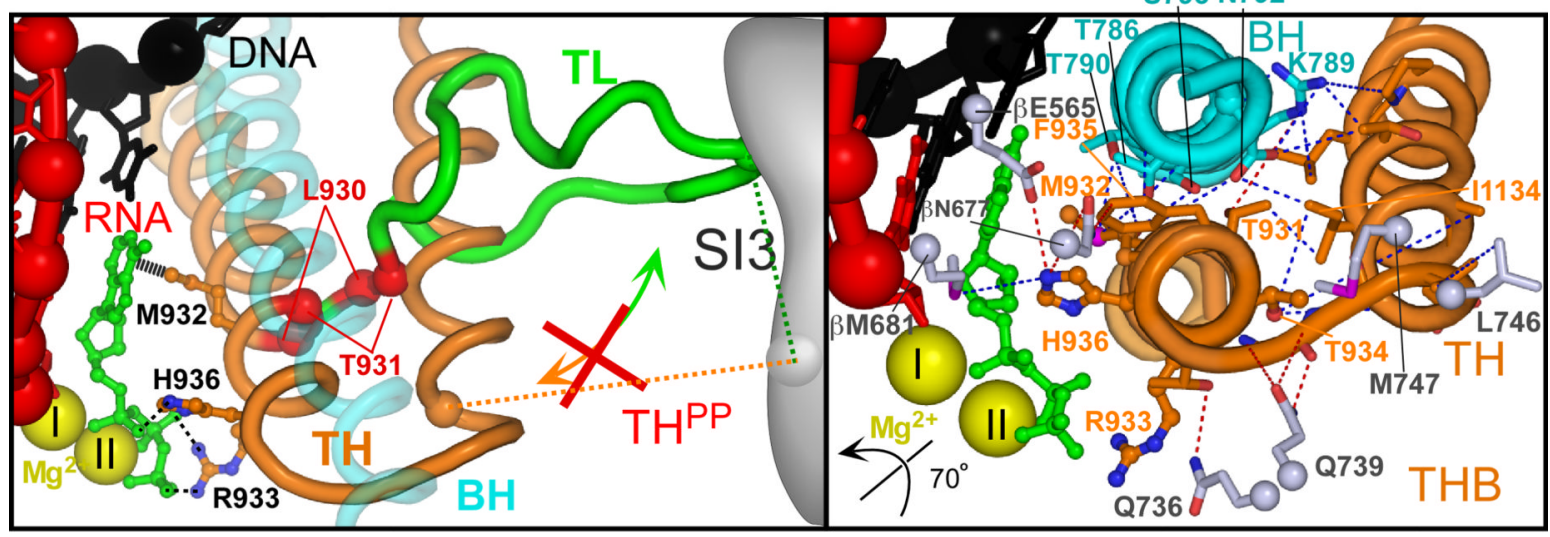

Fig. 1.

Nucleotidyl transfer and RNA hydrolysis reactions catalyzed by multisubunit RNAPs. (a) Nucleotidyl transfer (left) and RNA hydrolysis (right) reactions occur by $\mathrm{S}_{\mathrm{N}} 2$ nucleophilic attack with electron and proton transfer via a two-metal-mediated trigonal bipyramidal transition state, with reactants positioned in the $\mathrm{P}$ and A sites. Blue, electron path. Green, proton path. A:, general acid. B:, general base. For nucleotidyl transfer (left), apparent NTP $K_{\mathrm{m}}$ app and $V_{\max }$ app (forward reaction) and rates of reverse reaction at $1 \mathrm{mM}$ PPi are shown for wild-type RNAP (black) and a mutant RNAP blocked in TL folding (TH ${ }^{\mathrm{PP}}$, red)6,8,40. For hydrolysis (right), apparent $\mathrm{Mg}^{2+} \mathrm{II} K_{\mathrm{d}}$ app and $V_{\max }$ app $\left(\min ^{-1}\right)$ for RNA cleavage in a 2- 
nt-backtracked scaffold (Figs. 2 and 3) in the absence (black) or presence (purple) of GreB are shown.

(b) Structure of RNAP catalytic center and proposed TH/TL transitions. DNA template, nascent RNA, incoming NTP, $\mathrm{Mg}^{2+}$ ions, and the bridge helix are shown in black, red, green, yellow, and cyan, respectively. TH (orange) PDB ID 2O5J6; unfolded TL (green) PDB ID 1IW77. E. coliSI3 (gray) is depicted in an approximate location24 with flexible connection to TL/TH (dotted lines). (c) The extensive network of electrostatic (red dotted lines) and van der Waals and hydrogen bonding (blue dotted lines) interactions that stabilize the THB (view rotated $70^{\circ}$ relative to b). Key contacting residues outside the THB also are shown (light gray). 
a

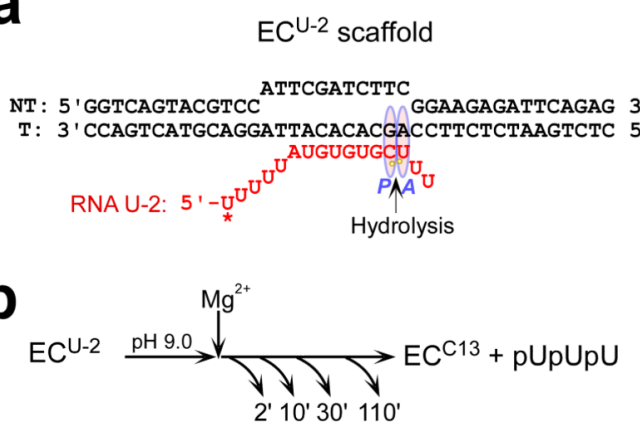

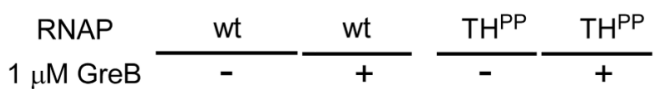

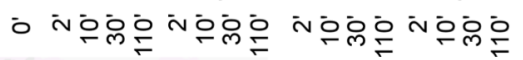

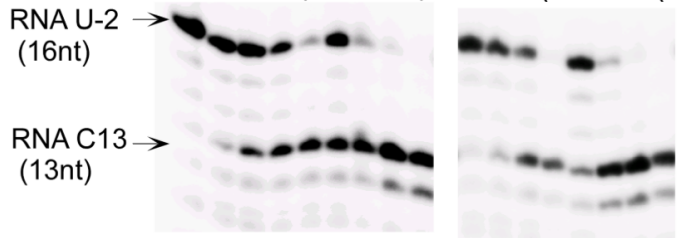

C

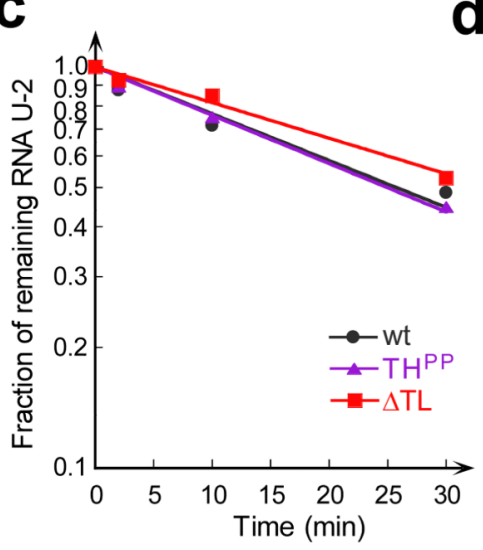

d

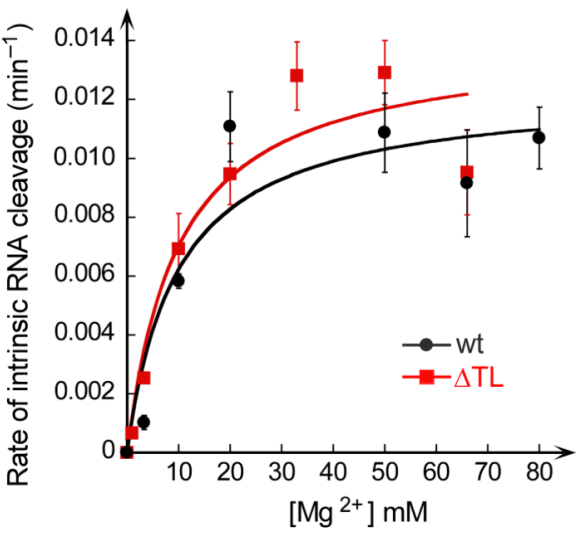

e

Estimated apparent $V_{\max }$ of intrinsic RNA cleavage and apparent $K_{d}$ for $\mathrm{Mg}^{2+} \mathrm{II}$

\begin{tabular}{ccc}
\hline RNAP & $V_{\max }^{\text {app }}\left(\mathrm{min}^{-1}\right)$ & $K_{\mathrm{d}}^{\mathrm{app}}$ for $\mathrm{Mg}^{2+} \mathrm{II}(\mathrm{mM})$ \\
\hline wt & $0.012 \pm 0.002$ & $10 \pm 6$ \\
$\Delta \mathrm{TL}$ & $0.014 \pm 0.002$ & $10 \pm 5$ \\
\hline
\end{tabular}

Fig. 2.

The TL is dispensable for intrinsic RNA hydrolysis on a locked scaffold. (a) Nucleic-acid scaffold. DNA and RNA are shown in black and red, respectively. Red asterisk, ${ }^{32} \mathrm{P}$ label. (b) Reaction scheme and electrophoretic separation of RNA products from a typical reaction at pH 9.0 and $20 \mathrm{mM} \mathrm{MgCl}_{2}$ by wild-type and $\mathrm{TH}^{\mathrm{PP}}$ RNAPs in the absence or presence of 1 $\mu \mathrm{M}$ GreB (Supplementary Methods). (c) Plot of the fraction of remaining RNA ${ }^{\mathrm{U}-2}$ against time by wild-type (black circles), $\mathrm{TH}^{\mathrm{PP}}$ (purple triangles), and $\Delta \mathrm{TL}$ (red squares) RNAPs in the absence of GreB. (d) Rates of intrinsic RNA ${ }^{\mathrm{U}-2}$ cleavage in the presence of $1-80 \mathrm{mM}$ $\mathrm{Mg}^{2+}$ and no GreB by wild-type (black circles) and $\Delta \mathrm{TL}$ (red squares) RNAPs. Error bars in all figures represent standard deviations from three or more replicates. (e) Estimated apparent $V_{\max }$ of intrinsic RNA cleavage and apparent $K_{\mathrm{d}}$ for $\mathrm{Mg}^{2+} \mathrm{II}$ derived from the curve-fitting in (d). 


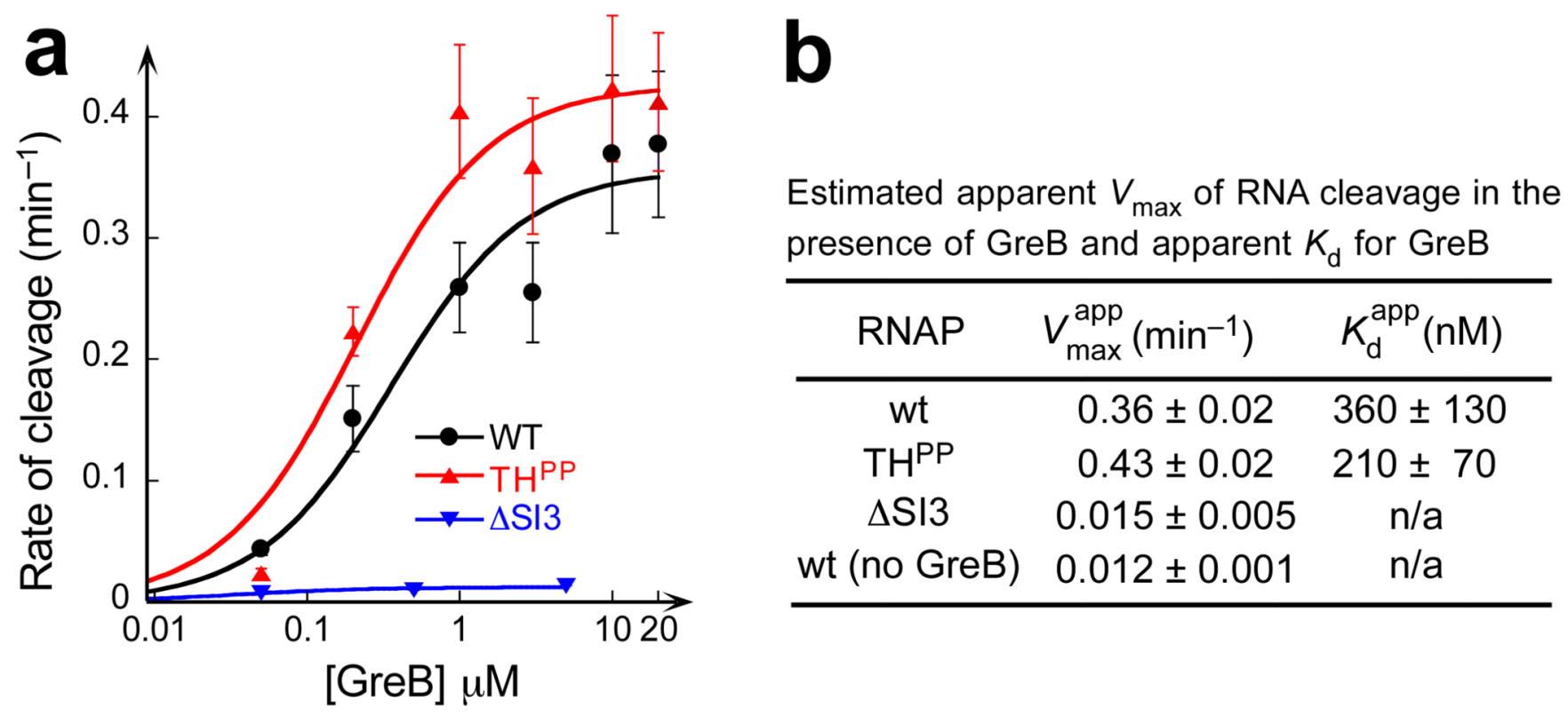

Fig. 3.

GreB requires SI3, but not TL folding, to stimulate transcript hydrolysis. (a) Transcript cleavage rates with $50 \mathrm{nM}$ to $20 \mu \mathrm{M}$ GreB by wild-type (black circles), $\mathrm{TH}^{\mathrm{PP}}$ (red triangles), and $\Delta$ SI3 (blue triangles) RNAPs. Hyperbolic fits were applied to wild-type and $\mathrm{TH}^{\mathrm{PP}}$ curves. (b) Estimated apparent $V_{\max }$ of GreB-stimulated RNA cleavage and apparent $K_{\mathrm{d}}$ for GreB to backtracked complexes. Kinetic parameters were derived from curve-fitting in (a) and Fig. 2d. 

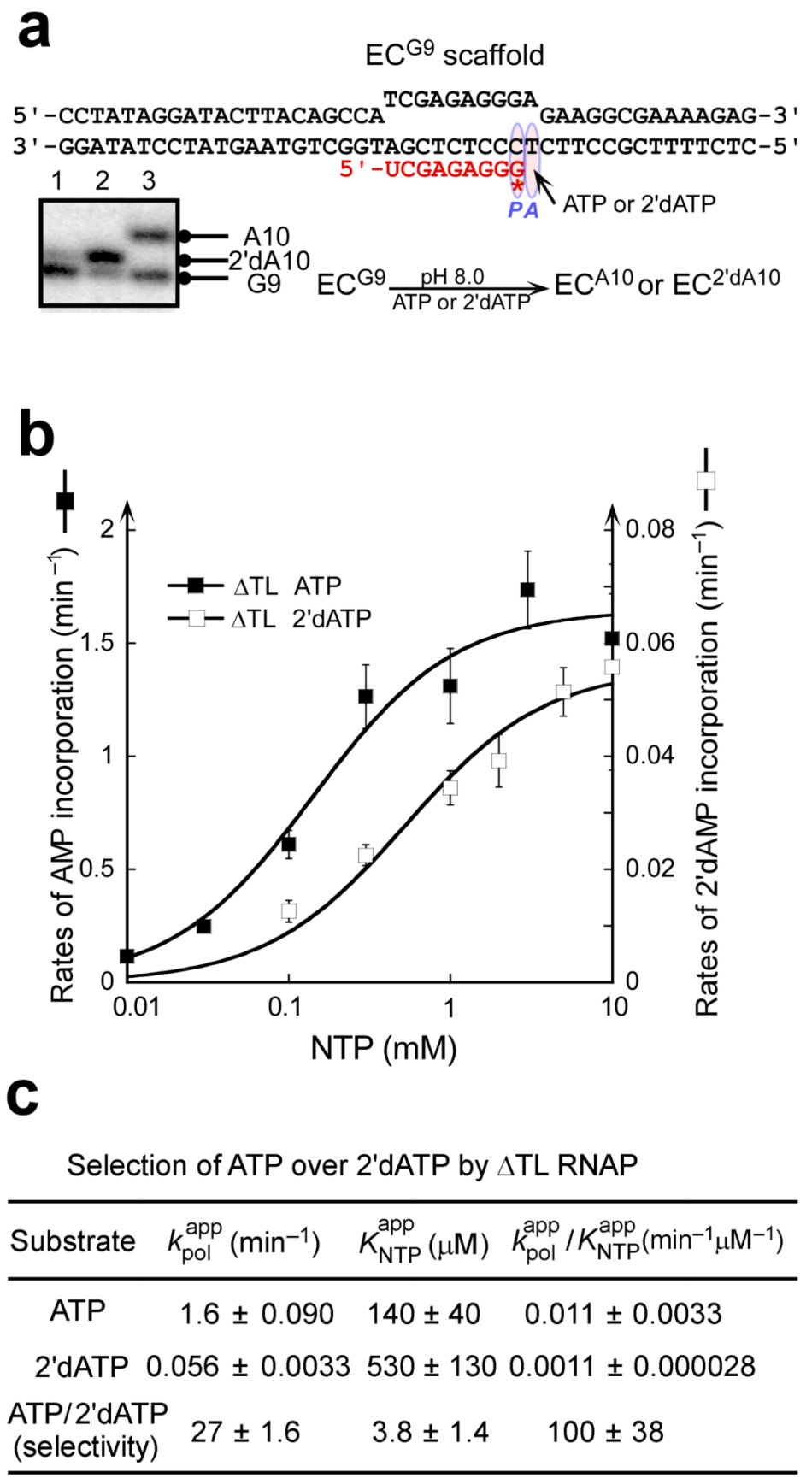

Fig. 4.

Significant discrimination against $2^{\prime}$ dNTPs is retained in $\triangle$ TL RNAP. (a) Nucleic-acid scaffold used to measure discrimination between ATP and $2^{\prime}$ dATP and reaction scheme. Red asterisk, location of $\left[\mathrm{a}^{-}{ }^{32} \mathrm{P}\right] \mathrm{GMP}$. The gel panel illustrates the distinct mobility of $\mathrm{RNA}^{\mathrm{A} 10}$ (incorporated AMP, lane 3) and RNA ${ }^{2} \mathrm{dA} 10$ (incorporated 2' dAMP, lane 2). (b) Selection of ATP over $2^{\prime}$ dATP by $\triangle$ TL RNAP. Reaction rates are plotted versus the concentration of ATP (filled squares; left y axis) or 2' dATP (hollow squares; right y axis; Supplementary Methods). Error bars represent standard deviations from 3 or more independent measurements. (c) $k_{\mathrm{pol}}$ app, $K_{\mathrm{d}}^{\text {app }}$, and selectivity $\left(k_{\mathrm{pol}}{ }^{\mathrm{app}} / K_{\mathrm{d}}{ }^{\text {app }}\right) 2$ for ATP and 
$2^{\prime}$ dATP by $\triangle$ TL RNAP were derived by hyperbolic curve-fitting weighted by standard deviation as depicted in (b), with errors propagated from standard error of the hyperbolic curve-fitting parameters $\left(R^{2}=0.967\right.$ and 0.959 for ATP and $2^{\prime}$ dATP curves, respectively; Supplementary Methods). 


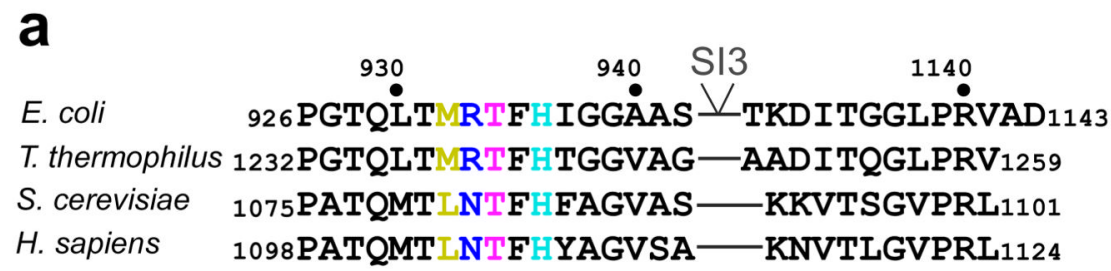

b

Pyrophosphorolysis

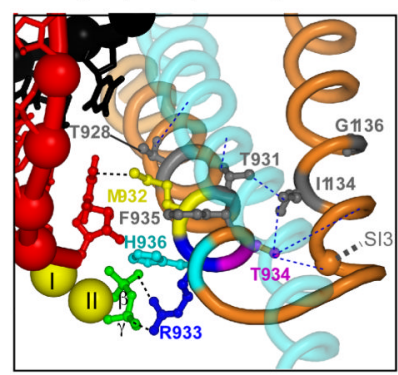

Pause escape

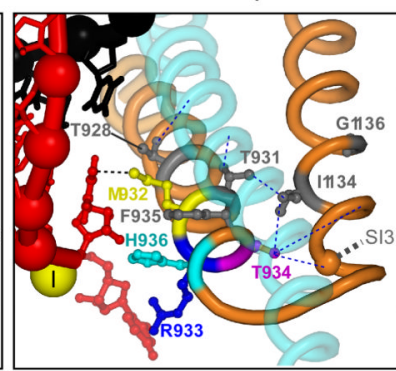

Rapid nucleotide addition (non-pause)

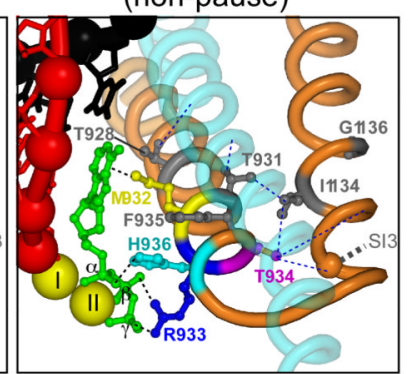

\section{C}

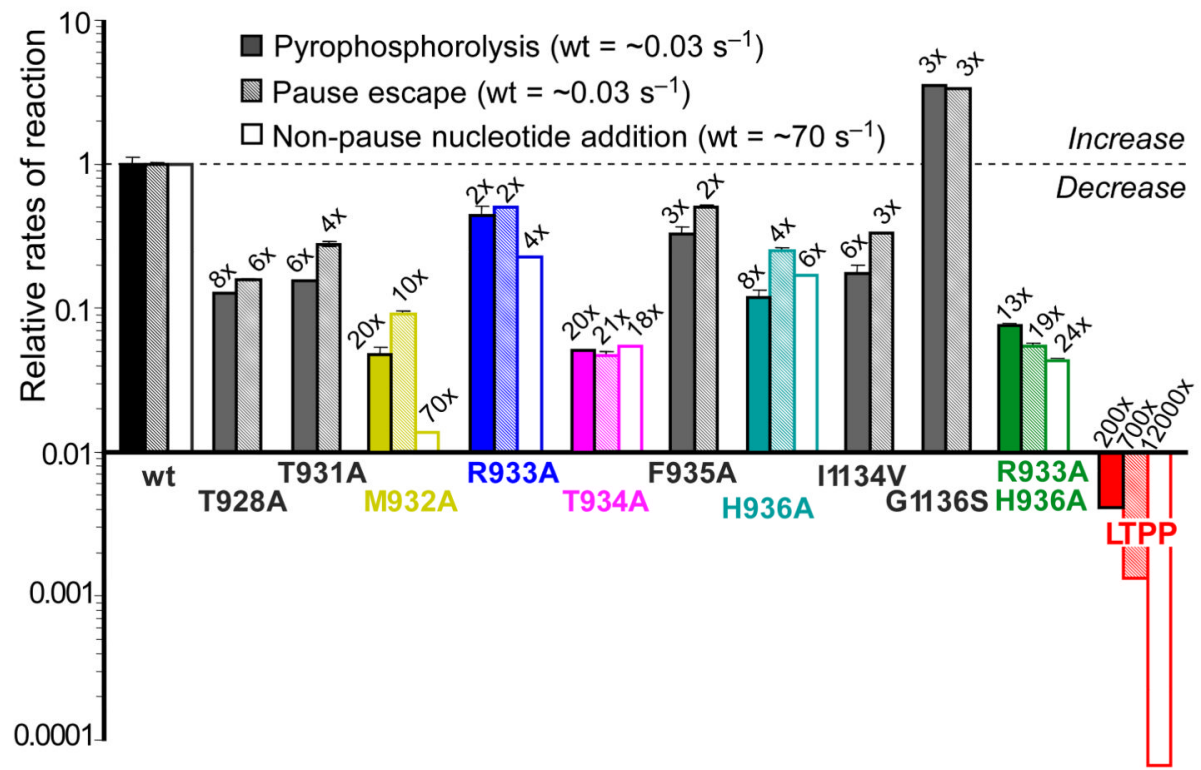

Fig. 5.

TL folding is similarly rate-limiting for rapid nucleotide addition, his pause escape, and pyrophosphorolysis. (a) Sequence alignment of TL sequences from representative RNAP or RNAPII enzymes. Residues are colored as in (b) and (c). (b) Active-site configurations for pyrophosphorolysis (left), pause escape (middle), and rapid nucleotide addition (non-pause; right). Location of selected TL residues that contact substrate NTP (M932, R933, and H936) or stabilize the THB (T931, T934, and I1134) are shown6. (c) Effects of single amino-acid substitutions of selected TL residues on pyrophosphorolysis (solid bars), pause escape (hatched bars), and non-pause nucleotide addition (open bars). Rates were normalized to 
those observed for the wild-type enzyme; numbers above the bars indicate fold difference with wild-type RNAP. Pause escape data for T928A, T931A, M932A, R933A, T934A, F935A, H936A, I1134V, and G1136S are from Toulokhonov et al. 200722. 


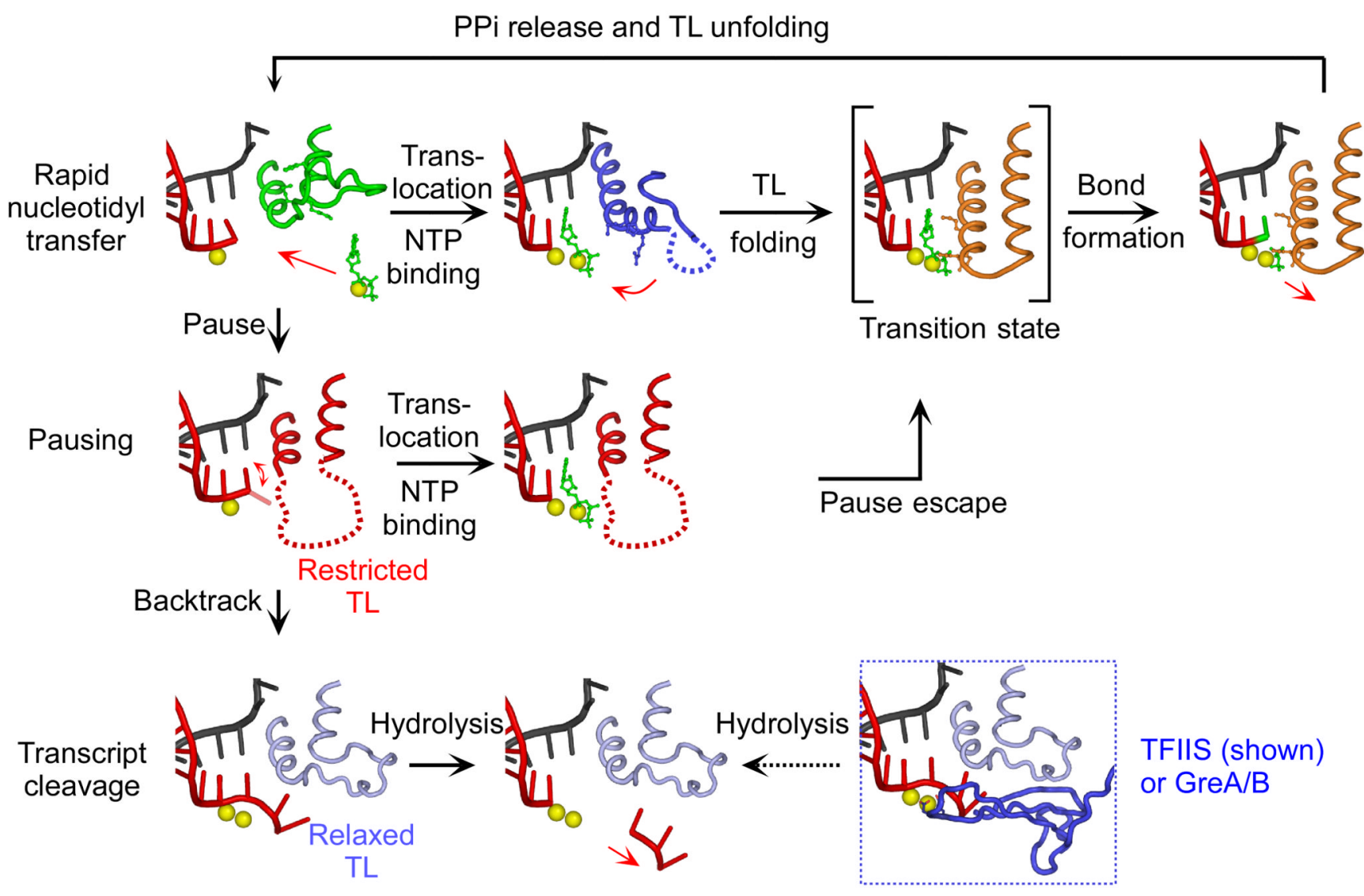

Fig. 6.

TL dynamics in nucleotidyl transfer, transcriptional pausing, and transcript cleavage.

Different TL conformations occur during nucleotidyl transfer (top row) and RNA hydrolysis (bottom row) reactions. During nucleotidyl transfer, a substrate (NTP or pyrophosphate) enters the active site while the TL is relaxed (green, PDB 1IW7)7, possibly inducing partial TL folding (blue, PDB 2O5I)40. Correct base-pairing of NTP to DNA template allows TH formation (orange, PDB 2O5J)6. After nucleotide addition, PPi exits the active site and TH unfolds into TL (top row). At non-backtrack pauses, the active site is transiently inhibited by a structural rearrangement (red) that allows or is caused in part by fraying of the RNA $3^{\prime}$ nt and that inhibits $\mathrm{TL} \rightarrow \mathrm{TH}$ folding 22 . The inhibited active site, nonetheless, appears able to translocate and to bind NTP22,33,34. To escape the pause, the TL must release from the restricted conformation (red, middle row). In contrast, backtracked complexes do not require TH formation, and the TL remains unfolded (light blue, PDB 1Y1V)12 during GreB- or TFIIS-stimulated RNA hydrolysis (bottom row). 